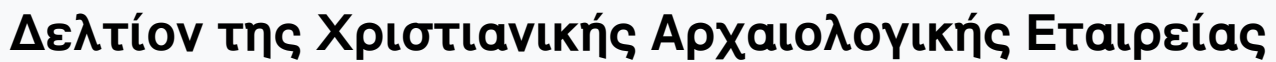

Tó 16 (1992)

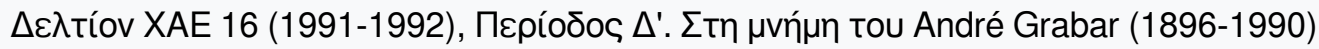

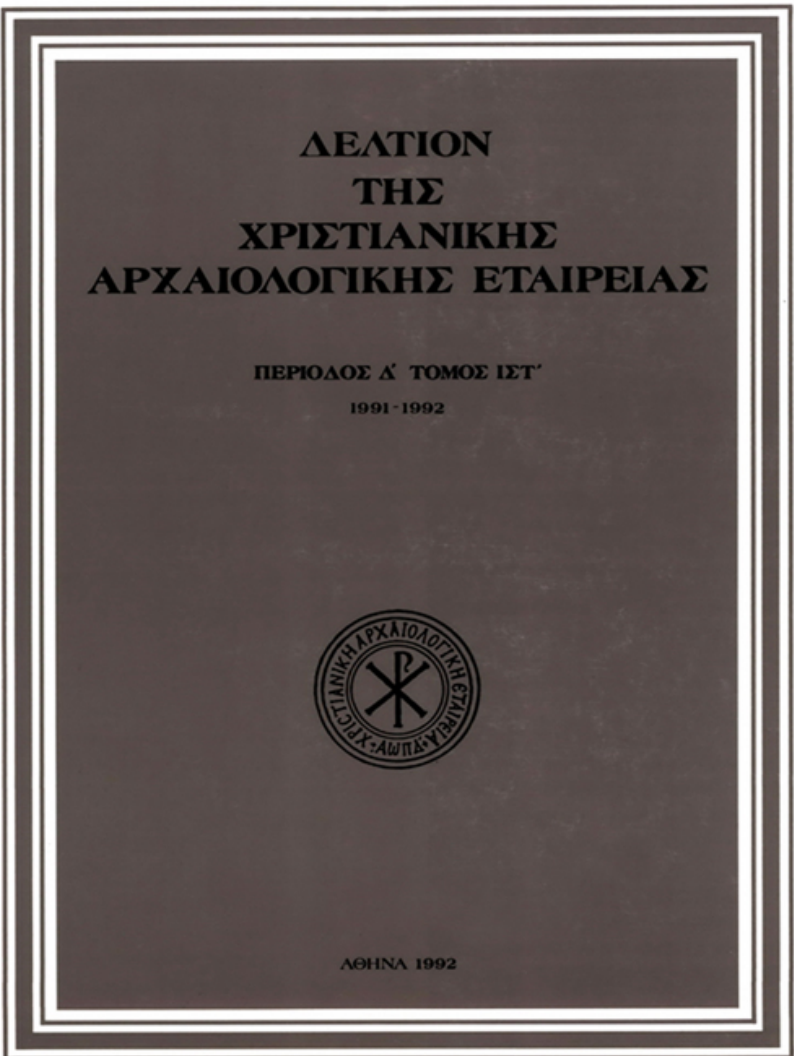

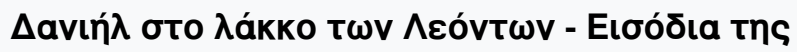

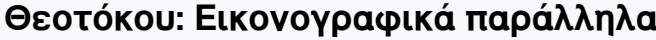

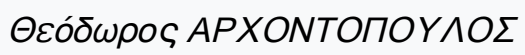

doi: $\underline{10.12681 / \text { dchae.1072 }}$

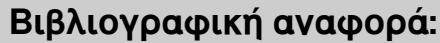

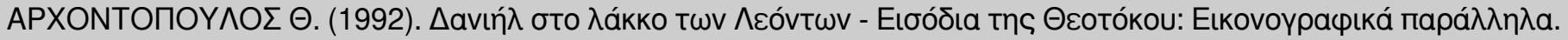

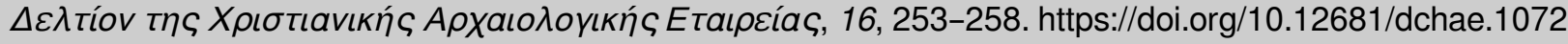




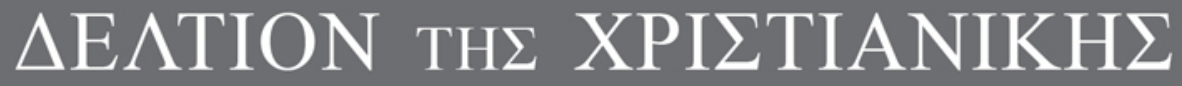 APXAIO $\Lambda$ OГIKH $\Sigma$ ETAIPEIA $\Sigma$}

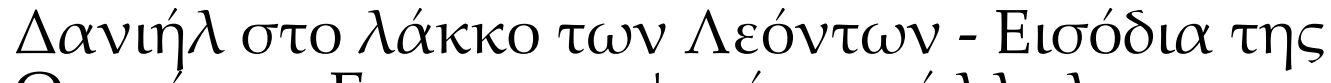

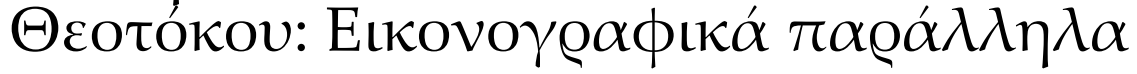

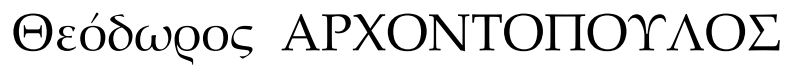

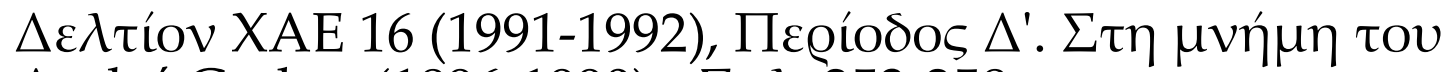
André Grabar (1896-1990)• $\Sigma \varepsilon \lambda$. 253-258

A@HNA 1992 


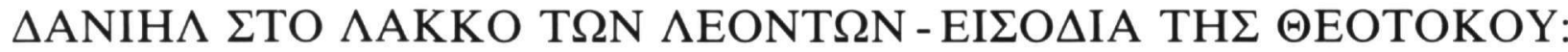 ЕIKONOГРАФIKА ПАРА $\Lambda \Lambda \mathrm{H} \Lambda \mathrm{A}^{1}$}

$\mathrm{H}$

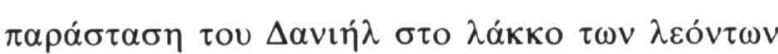

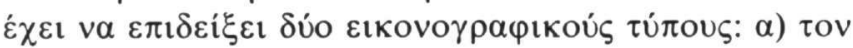

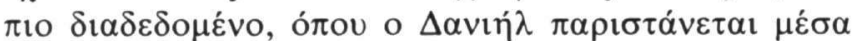

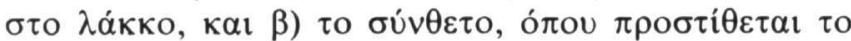

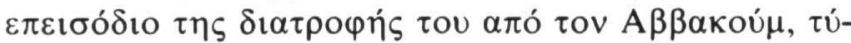

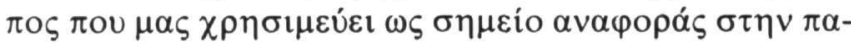

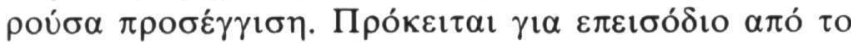

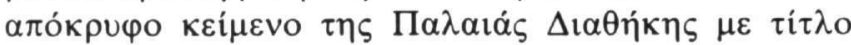

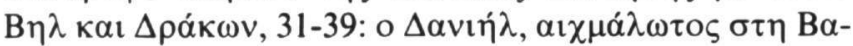

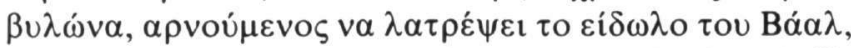

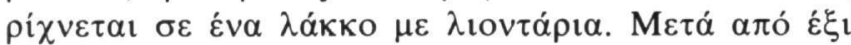

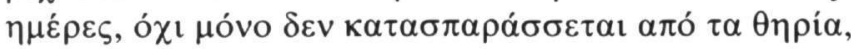

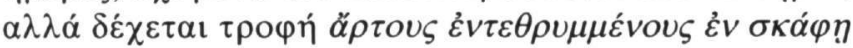

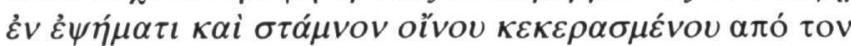

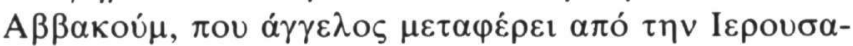
$\lambda \eta \dot{\mu} \sigma \tau \eta \mathrm{B} \alpha \beta \nu \lambda \dot{\omega} v \alpha$.

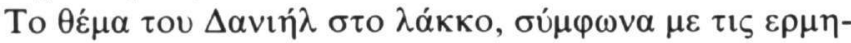

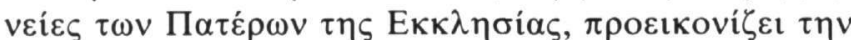

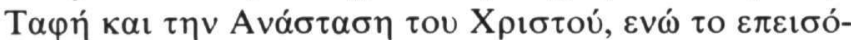

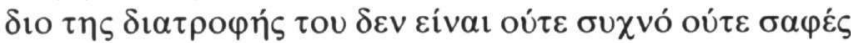

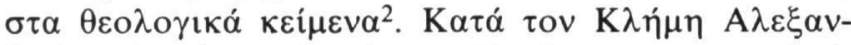

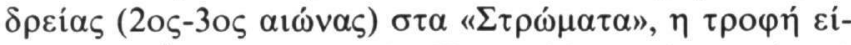

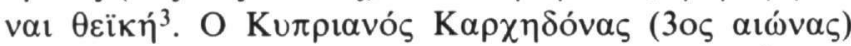

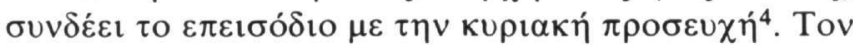

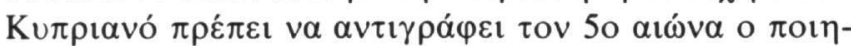

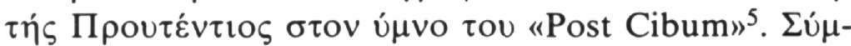

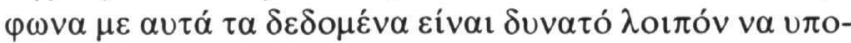

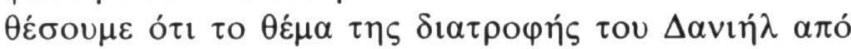

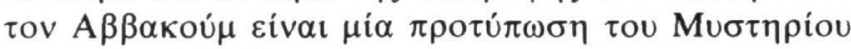

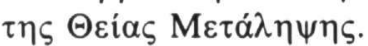

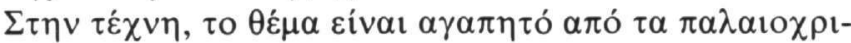

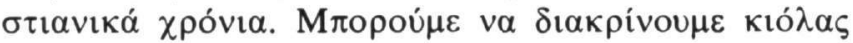

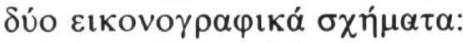

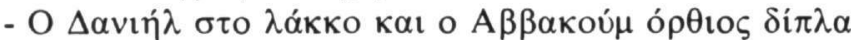
$\tau$ Tov $^{6}$.

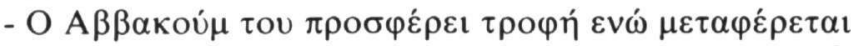

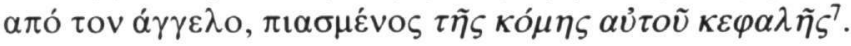

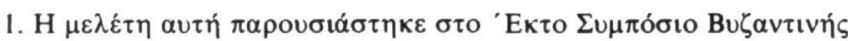

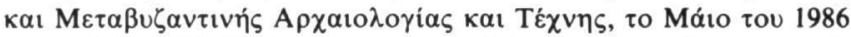

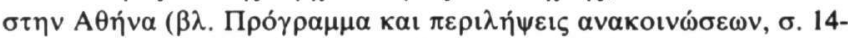
15). A mes vétérotestamentaires dans la peinture murale à l’époque des Palé-

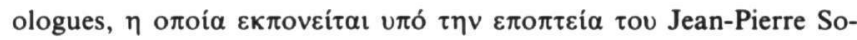
dini.

2. K. Künstle, Iconographie der christlichen Kunst 1, Freiburg i. Br. 1928, б. 18-19. L. de Bruyne, L'imposition des mains dans l'art chrétien ancien, RAC 20 (1943), $\sigma$. 191. J. Ra dova nović, Iconographija fresaka protezisa crkva svetih Apostola u Peći, ZLU 9-10 (1959), б. 105-114. Gordana Babić, Simbolicmo znacenje zivopisa u protezisn vetih Apostola u Peći, Zbornik zasite spomenica kulture 15

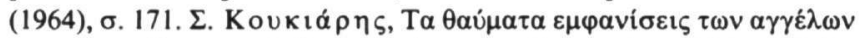

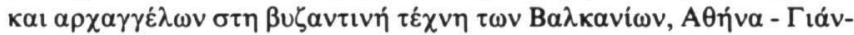
viva 1989, б. 143-144.

3. GCS, Clemens Alexandrinus, Berlin 1985, б. 77. SC, Série grecque,

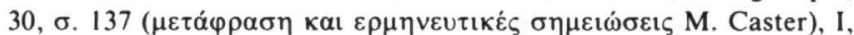

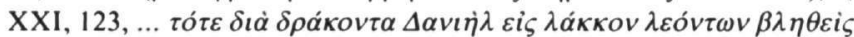

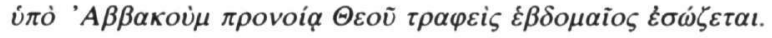

4. M. Jourdon, Cyprien de Cartage, Paris 1957, o. 114.

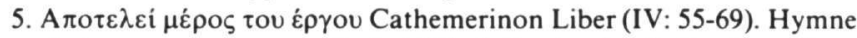
après le repas, Lavarenne, Paris 1945, Collection Budé, Prudence, I, $\sigma$. 22:

«....Cernit forte procul dapes inemptas, quas messoribus Ambacum propheta agresti bonus exhiberat arte, hujus caesariae manu prehensa, plenis, sicut, erat, gravem canistris suspensum rapit et vehit per auras. Tum raptus simul ipse prandiumque sensim labitur in lacum leonum, et quas tunc epulas gerebat offert. "sumas laetus" ait "libensque carpas, quae summus Pater angelusque Christi mittunt liba tibi sub hoc periclo"..

Sic nos nuberibus tuis refecti, largitor Deus omnium bonorum, grates reddimus et sacramus hymnos...".

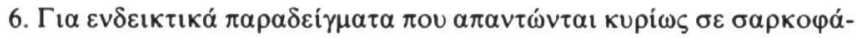

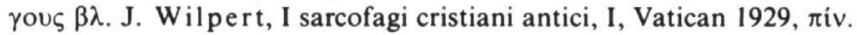
$131,136,177.4,187.2,206.2,208.5,208.9,208.10 \kappa \tau \lambda$

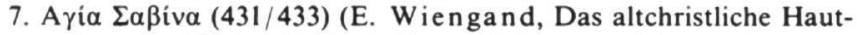
portal an der Kirche der St. Sabina auf dem aventinischen Hügel zu Rom, Trier 1900, б. 65-69. S. Tsuji, Les Portes de Sainte Sabine, Particularités de l'iconographie de l'Ascension, CahArch 13 (1962). G. Jeremias, Die Holztür der Basilika S. Sabina in Rom, Tübingen 1980, б. 68-72)

Пи $\tau \eta \varsigma$ Trier $\left(50 \varsigma \alpha_{1}\right.$.) (W.F. Volba ch, Elfenarbeiten der Spätantike und des frühen Mittelalters, Meinz 1952, 1976, $\alpha \rho t \theta .167,162)$. Avó $\gamma \lambda \nu \varphi o$

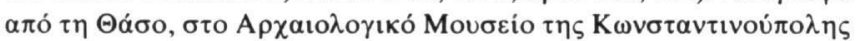
(5o $\alpha_{\text {l.) }}$ (H. Peirce - R. Tyrel, Elephant-Tamersilk, 8th Century, DOP 2 (1941), عıк. 12. A. G rabar, Sculptures byzantines de Constan-

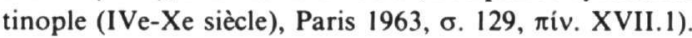

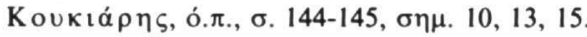




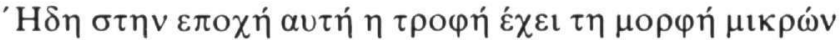

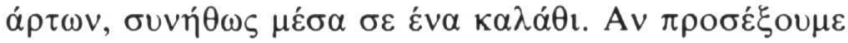

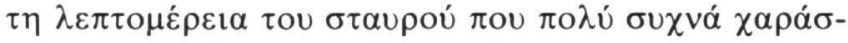

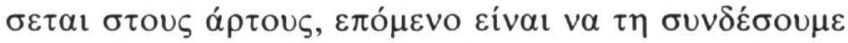

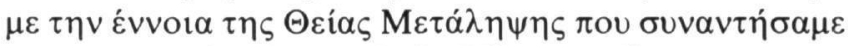

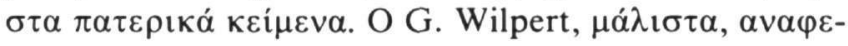

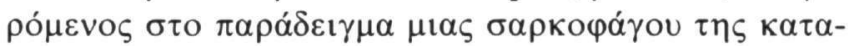

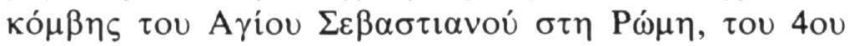

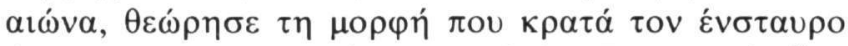
$\alpha \rho \tau o ~ \sigma \alpha v \pi \rho \circ \sigma \omega \pi$ o

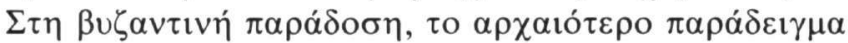

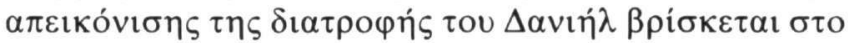

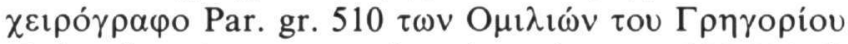

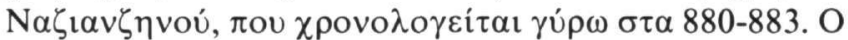

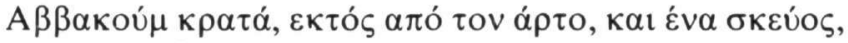

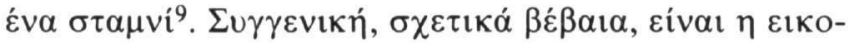

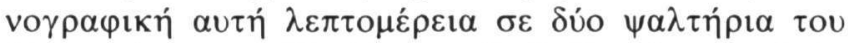

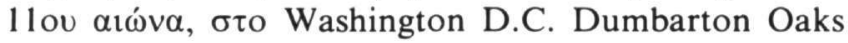

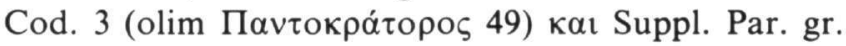

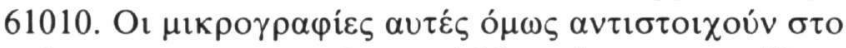

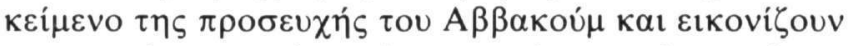

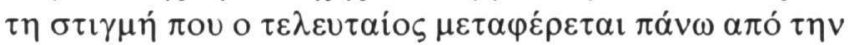

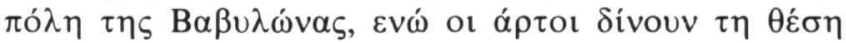

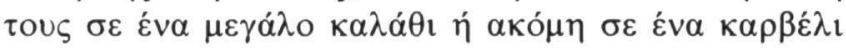

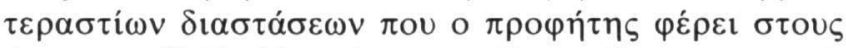

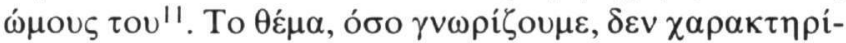

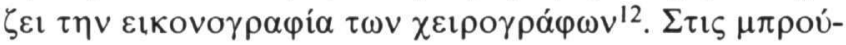

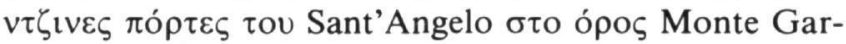

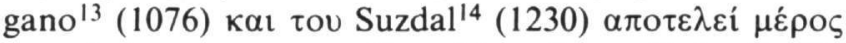

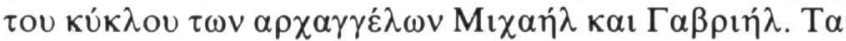

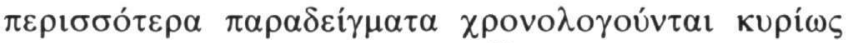

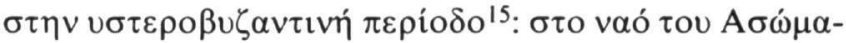

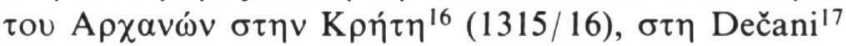

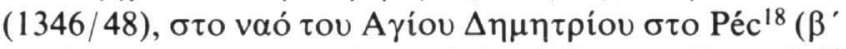

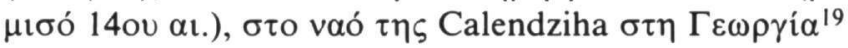

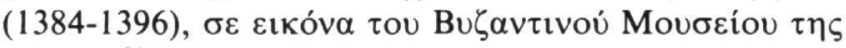
A $\theta \dot{v} v \alpha \varsigma^{20}(14 \mathrm{o \zeta} \alpha \mathrm{l}$.).

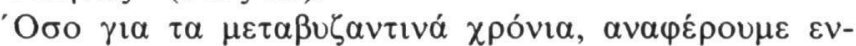

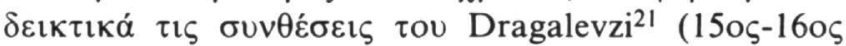

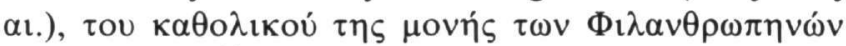

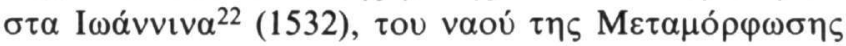

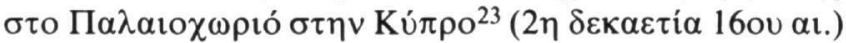

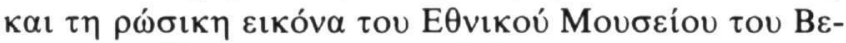

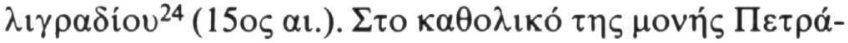

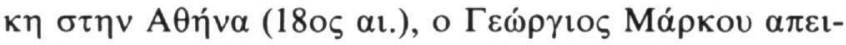

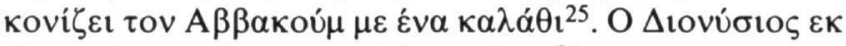

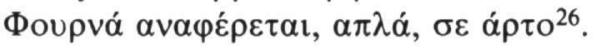

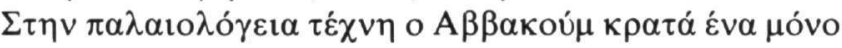

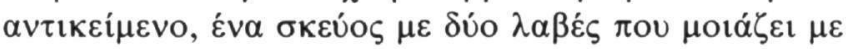

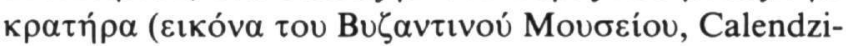

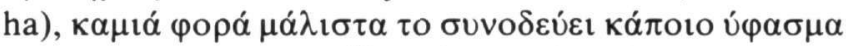

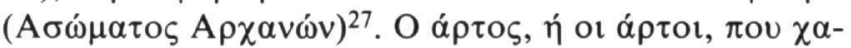

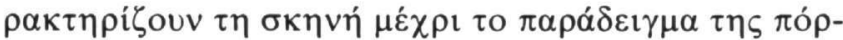

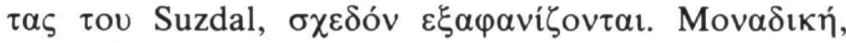

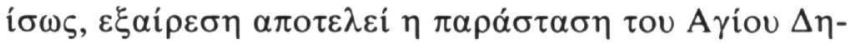
$\mu \eta \tau$ píov tov Peć, ó

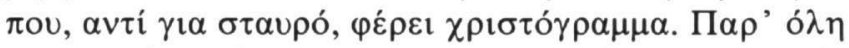

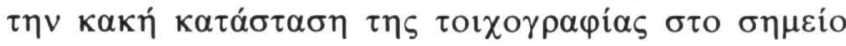

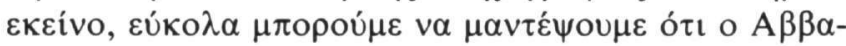

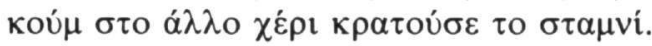

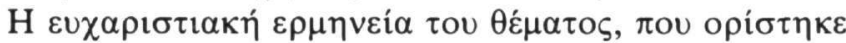

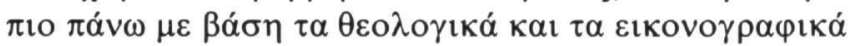

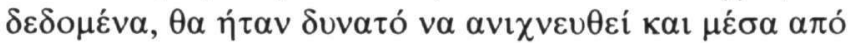

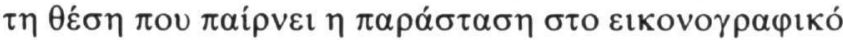

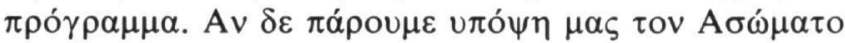

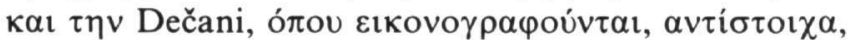

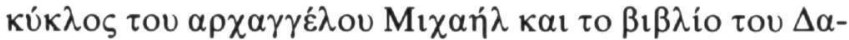

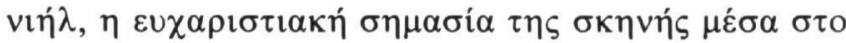

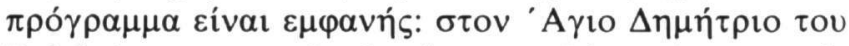

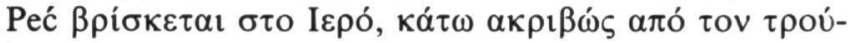

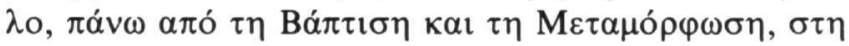

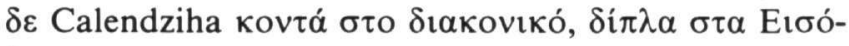
$\delta i \alpha$.

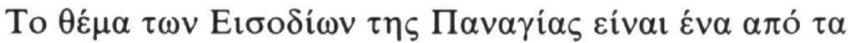

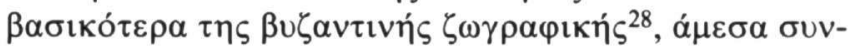

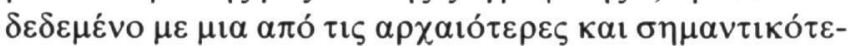

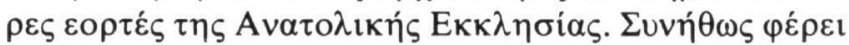

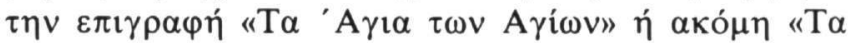

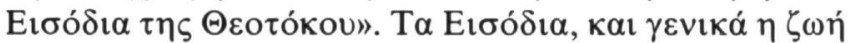

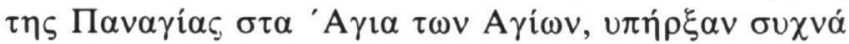

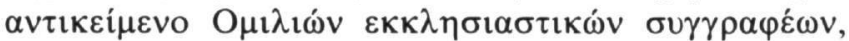

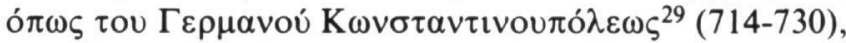

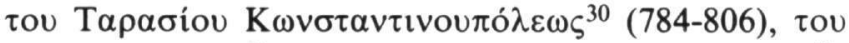

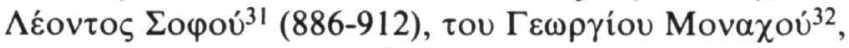

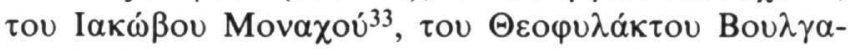

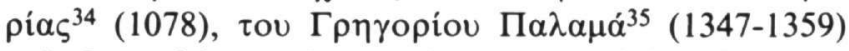

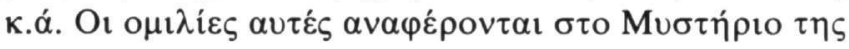

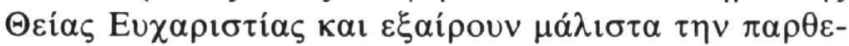

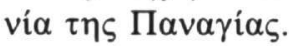

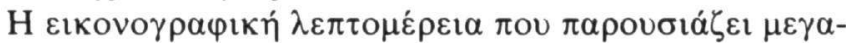

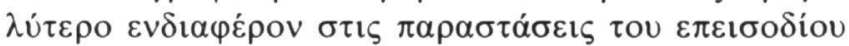

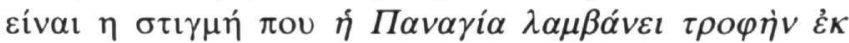

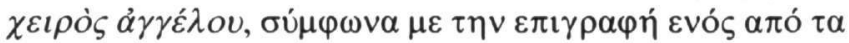

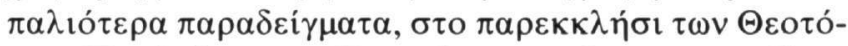

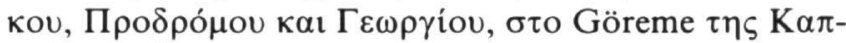

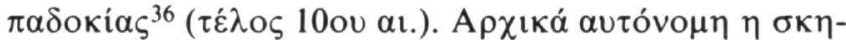

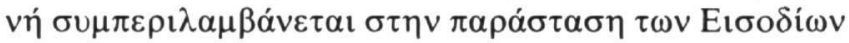

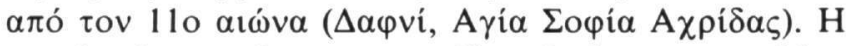

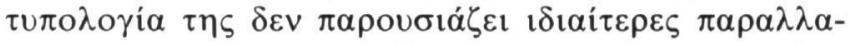

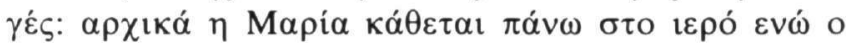

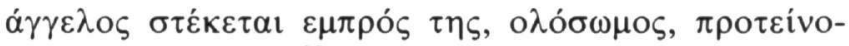

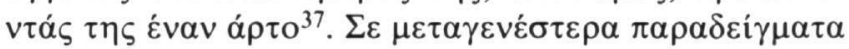

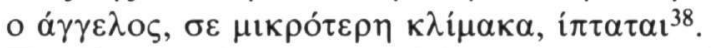

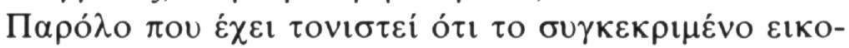




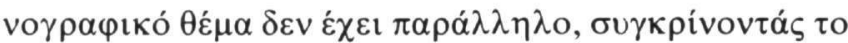

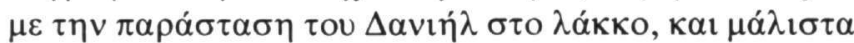

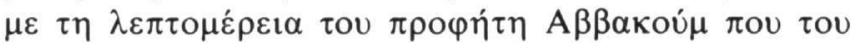

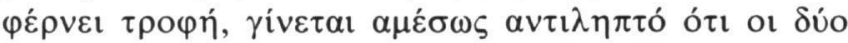

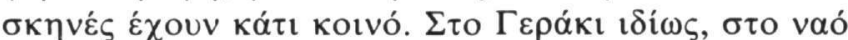

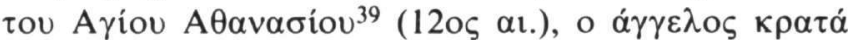

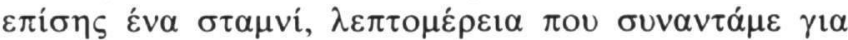

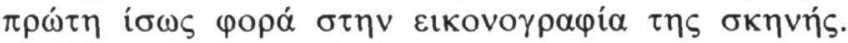

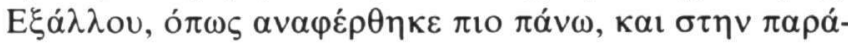

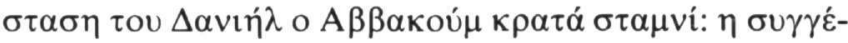

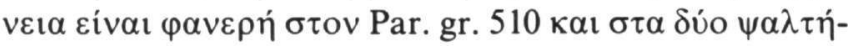

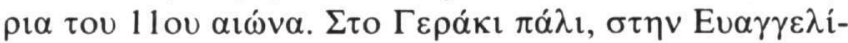

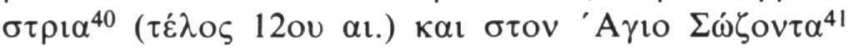

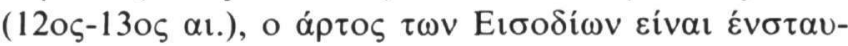

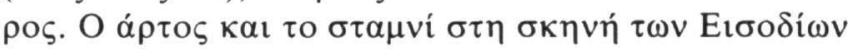

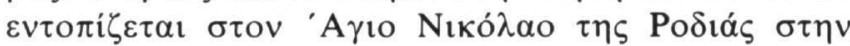

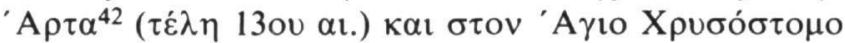

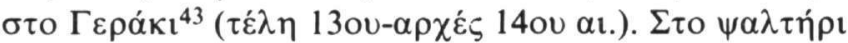

8. J. Wilpert, Fractio Panis-Die älteste Darstellung des eucharisti-

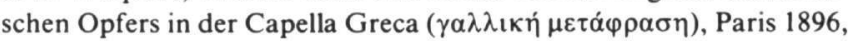
$\sigma .66$. O $\mathrm{i} \delta \mathrm{\imath}$ o, Il simbolismo eucaristico del cibo di Daniele nelle fossa dei leoni, Atti della Pontificia Accademia Romana di Archeologia Cristiana, Serie III, Rendiconti 9 (1933-1934), б. 89-94.

9. K. Weitzmann, The Ode Picture of the Aristocratic Psalter Re-

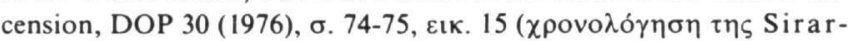
pie Der Nersessian, The Illustrations of the Homilies of Gregory of

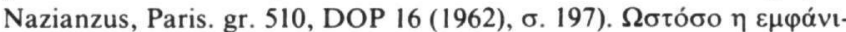

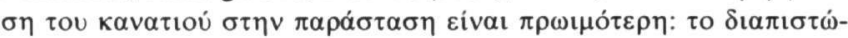

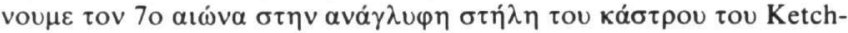

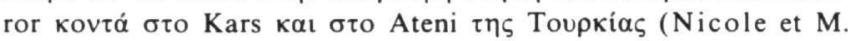
Thierry, A propos de quelques monuments chrétiens du vilayet de Kars (Turquie), Revue des Etudes Arméniennes - nouvelle série VIII (1971), б. 197-198, عเк. 15, 16).

10. K. Weitzmann, The Joshua Roll, Princeton 1947, عıк. 86, 157. C. Astruc, Un psautier byzantin à frontispices: Le suppl. gr. 610 , CahArch 3 (1958), б. 106-113, عıк. 3, 4. Sirarpie Der Nersessian, A Psalter and New Testament Manuscript in Dumbarton Oaks, DOP 19 (1965), б. 17, عıк. 13, 14. A. Cutler, The Aristocratic Psalters in Byzantium, Paris 1984, б. 72, 74, عıк. 262, 329.

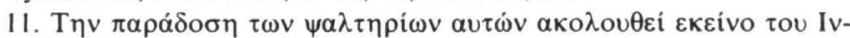

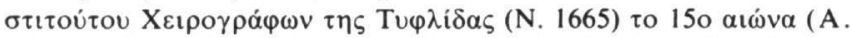
Alpago-Novello, V. Beridze, Jacqueline Lafontaine-Dosogne, Art and Architecture in Medieval Georgia, Louvain 1980, عıк. $69)$.

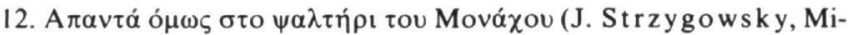
niaturen des serbischen Psalters, Wien 1906, $\pi$ iv. 49).

13. Margaret English-Frazer, Church and the Gates of Paradise: Byzantine Bronze Doors in Italy, DOP 27 (1973), عıк. 23.

14. A. N. Ovchinnikov, Golden Gates in Suzdal, Moscow 1978,

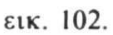

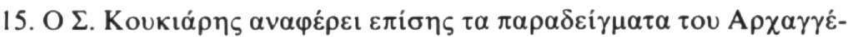

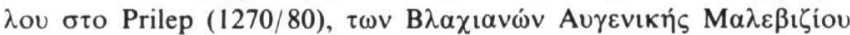

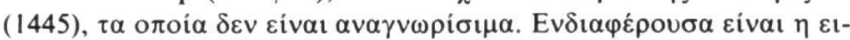

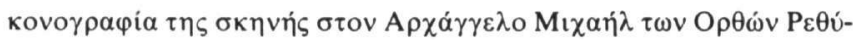

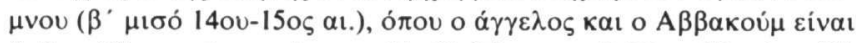

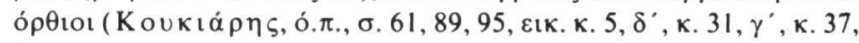
$\left.\beta^{\prime}\right)$.

16. M. Chatzidakis, Rapports entre la peinture de la Macedoine et

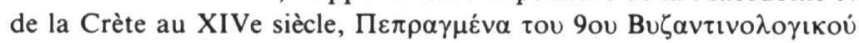

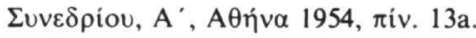

17. V. R. Petković - G. Bosković, Dečani (Les peintures), Belgrade 1941, rív. CCLXVIII.

18. V. R. Petković, La peinture serbe du moyen-âge, Belgrade 1954, $\pi$ iv. XCI.

19. H. Belting, Le peintre Manuel Eugenikos de Constantinople, en Géorgie, CahArch 28 (1979), $\sigma .103$. Doula Mouriki, Reflection of Constantinopolitan Style in Georgian Monumental Painting, XVI. Internationaler Byzantinistenkongress, Wien 1981, JÖB 31/2, Akten $1 / 2, \sigma .750, \varepsilon เ \kappa .47$.

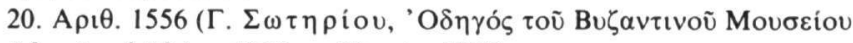

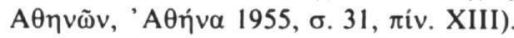

21. Assen Tshi Lingirou, Die Kunst des christlichen Mittelalters in Bulgarien, 4. bis 18. Jahrhundert, München 1979, б. 77.

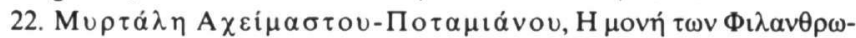

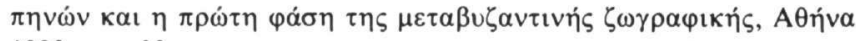
1983 , عเк. $32 \alpha$.

23. A. and Judith Stylianou, The Painted Churches of CyprusTreasures of Byzantine Art, London 1985, б. 273.

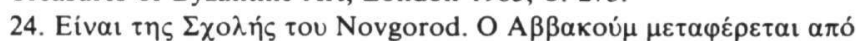

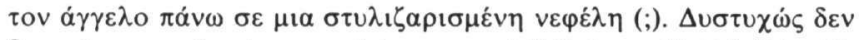

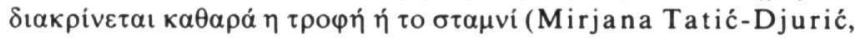
Danil medju lavovima-Jedan primerak novgorodske sli karske skole XV veka, Zbornik Radova Narodnog Muzeja II (1959), єıк. 1.4).

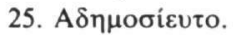

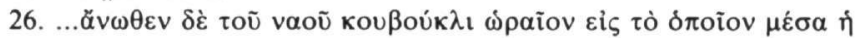

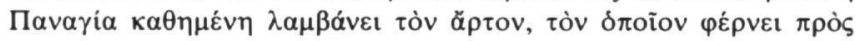

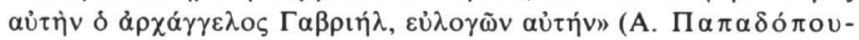

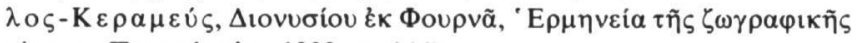

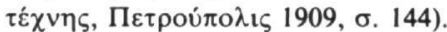

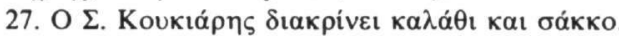

28. Jacqueline Lafontaine-Dosogne, Iconographie de l'enfance de la Vierge dans l'empire byzantin et en Occident, I, Bruxelles 1964, б. 136-167.

29. PG 98, 309-320.

30. PG 98, 1481-1500.

31. PG 167, 12-21.

32. PG $100,1401-1420$.

33. PG $127,600-632$.

34. PG 126, 129-144.

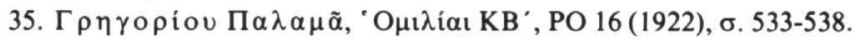
36. M. Restle, Die byzantinische Wandmalerei in Kleinasien, Recklinghausen 1967, ع1к. 125. Guillaume de Jerphanion, Les églises

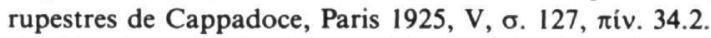

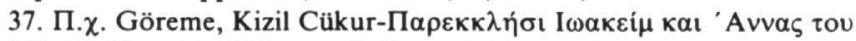
$850 / 60$ (N. et M. Thierry, Eglise de Kizil-Tcoukour, chapelle iconoclaste, chapelle de Joachim et Anne, MonPiot 50 (1958), $\sigma .105-146$.

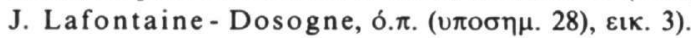

38. Il Menologio di Basilio II (fascimile II, tavole), Torino 1907, $\varphi$. $252 \mathrm{~b}$.

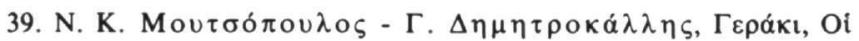

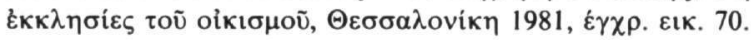

40. 'О.

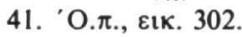

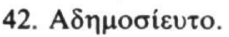

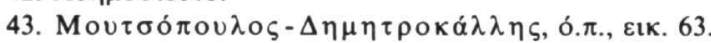




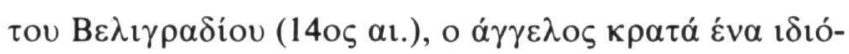

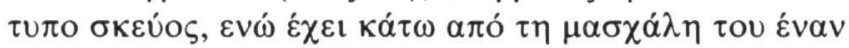
$\alpha \dot{\rho} \tau$ о $\sigma \varepsilon \mu \varepsilon \dot{\varepsilon} \gamma \varepsilon \theta \varsigma$ o $\kappa \alpha \rho \beta \varepsilon \lambda$ เoú.

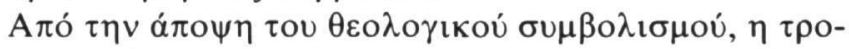

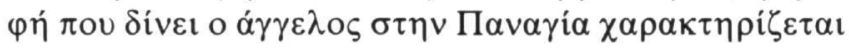

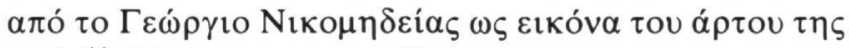

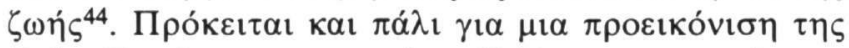

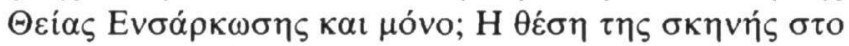

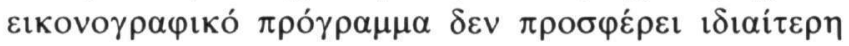

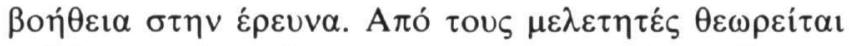

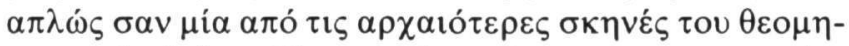

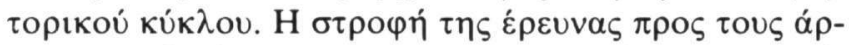

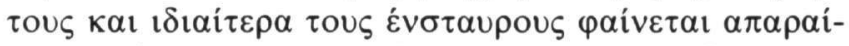

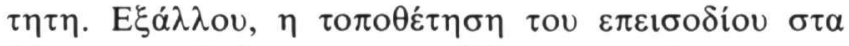

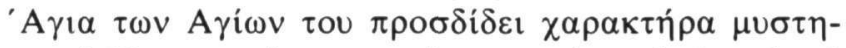

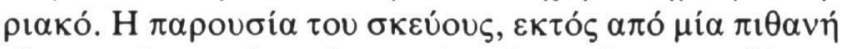

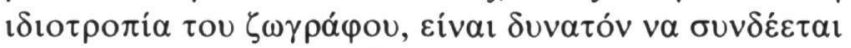

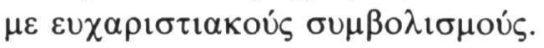

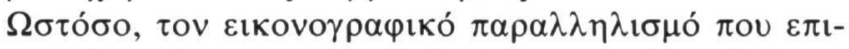

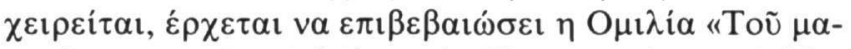

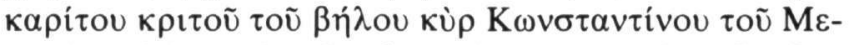

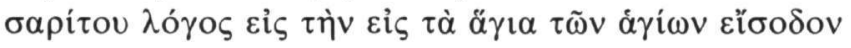

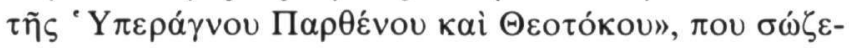

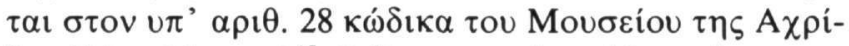

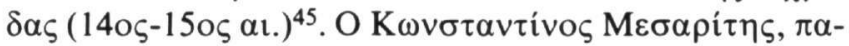

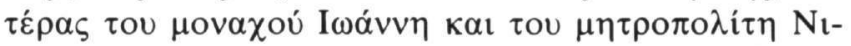

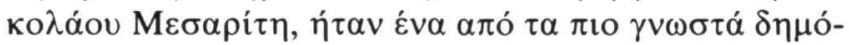

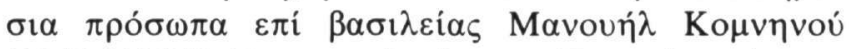

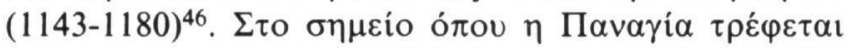

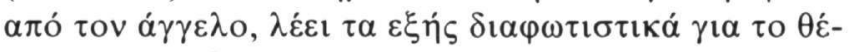

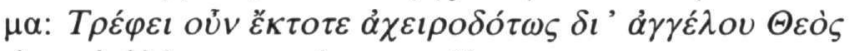

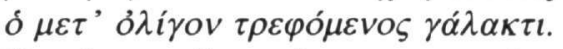

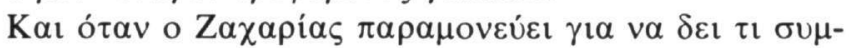

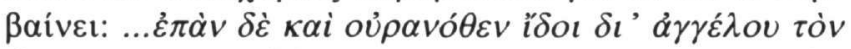

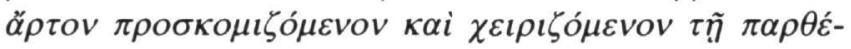
$v \omega \ldots$

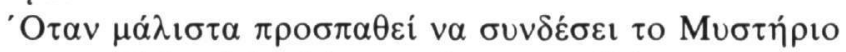

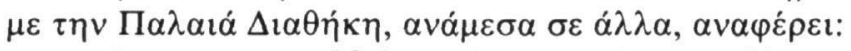

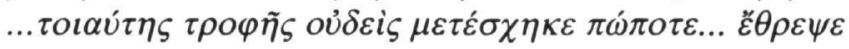

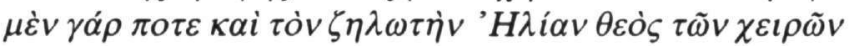

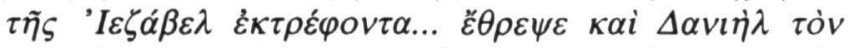

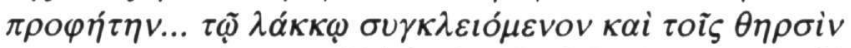

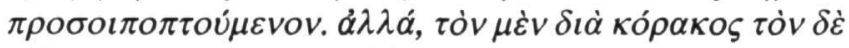

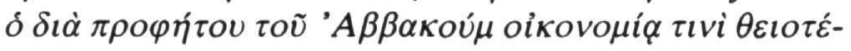

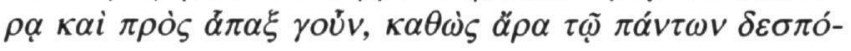
$\tau \eta \ldots$

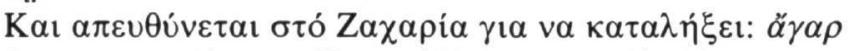

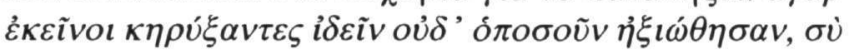

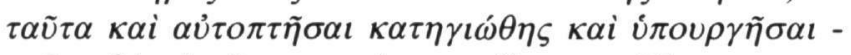

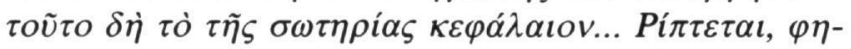

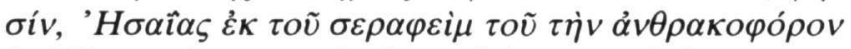

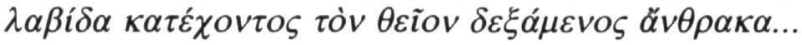

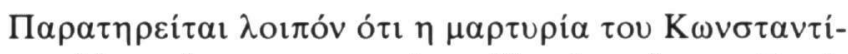

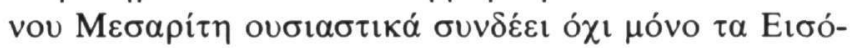

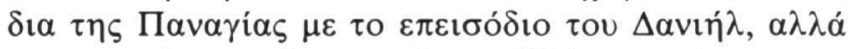

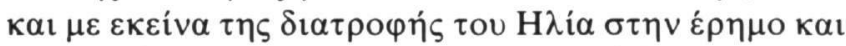

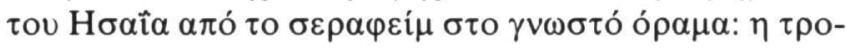

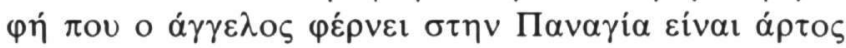

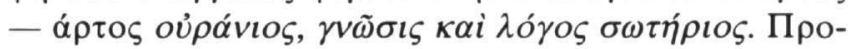

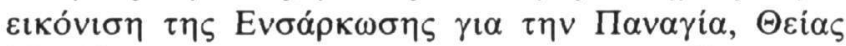

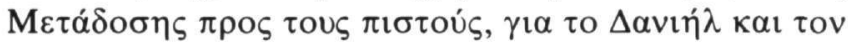
Hiia.

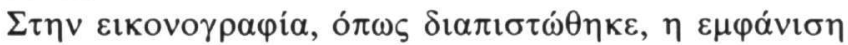

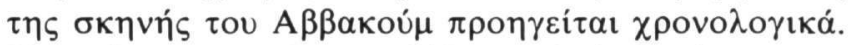

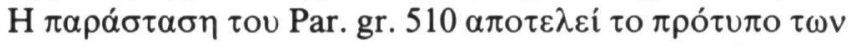

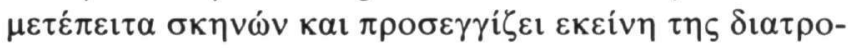

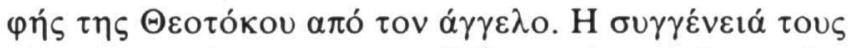

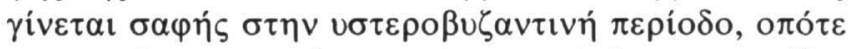

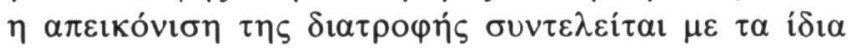

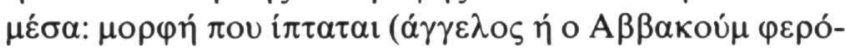

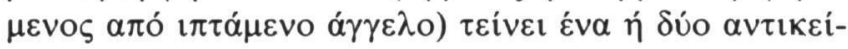

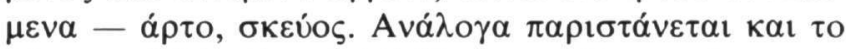

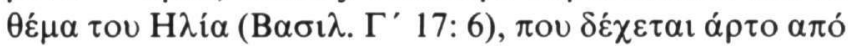

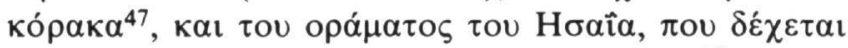

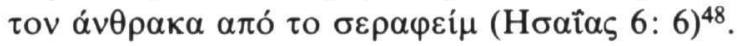

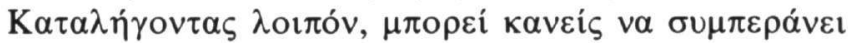

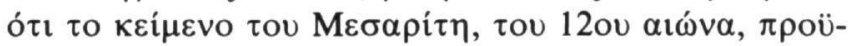

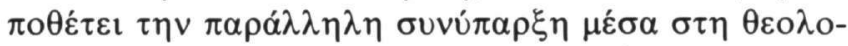

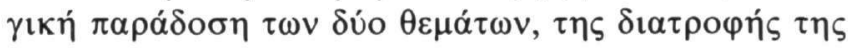

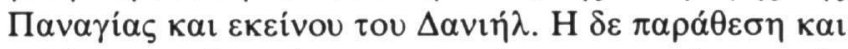

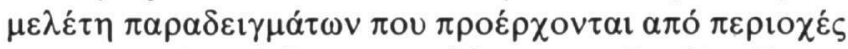

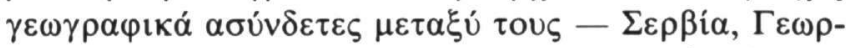

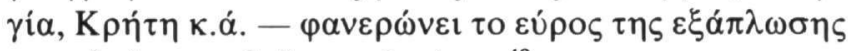

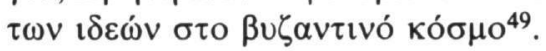

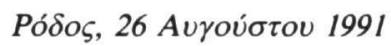

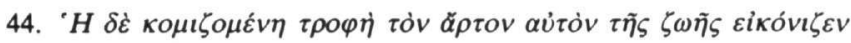
(PG 100, 1443).

45. F. Halkin, Inédits byzantins d'Ochrida, Candie et Moscou, SubsHag 38 (1963), б. 46-49.

46. H. G. Beck, Kirche und theologische Literatur im byzantinischen Reich, München 1959, б. 665-667.

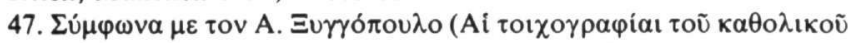

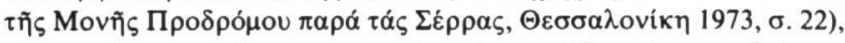

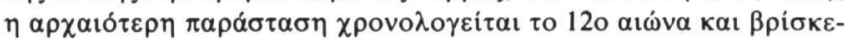

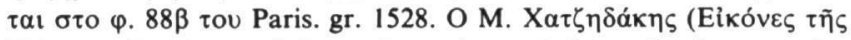

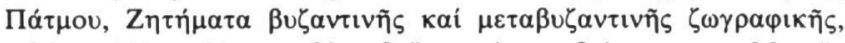

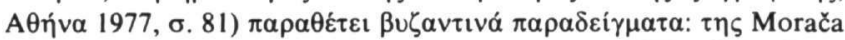
(1272) (V. Djurić, La peinture murale serbe au XIIIe siècle, L'art

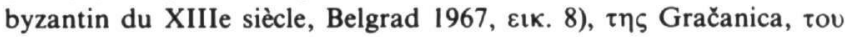

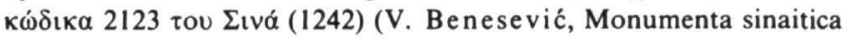




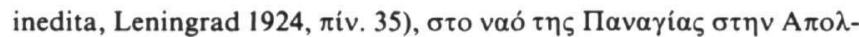

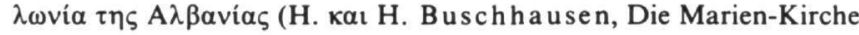
von Apollonia in Albanien, Byzantina Vindobonensia VIII (1967), $\sigma$.

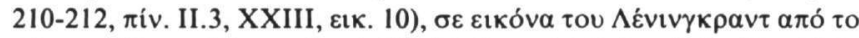

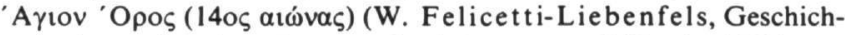
te der byzantinischen Ikonenmalerei, Lausanne 1956, $\pi$ iv. 100b).

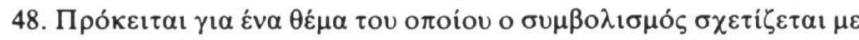

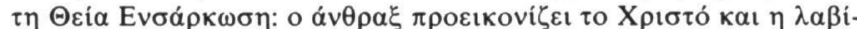

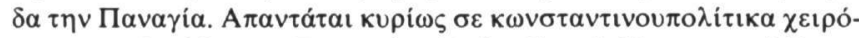

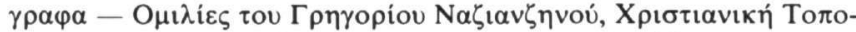

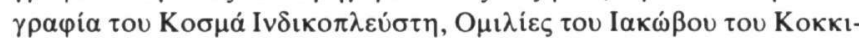

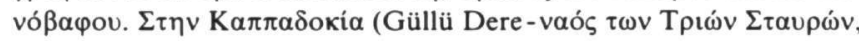

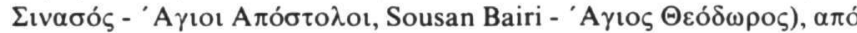

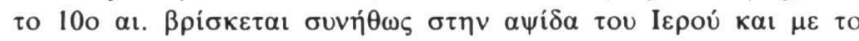

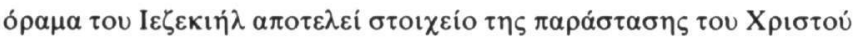
$\sigma \varepsilon \delta \delta ́ \xi \alpha$ (Jacqueline Lafontaine-Dosogne, Théophanies auquelles participent les prophètes dans l'art byzantin après la restaura-

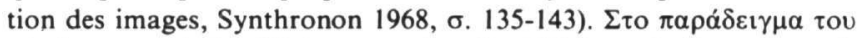

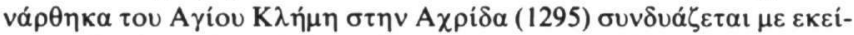

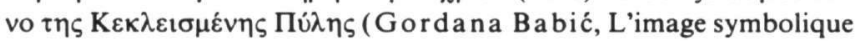
de la "Porte Fermée" à Saint-Clément d'Ohrid, Synthronon 1968, $\sigma .145-147)$

49. O E. Mâle (L'art religieux du XIIIe siècle en France, Paris 1958, $\sigma$.

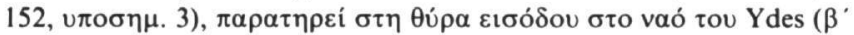

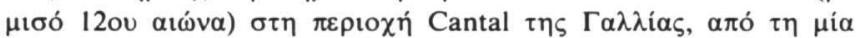

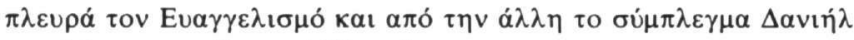

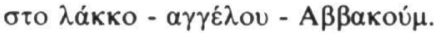




\section{Théodore Archontopoulos}

\section{DANIEL DANS LA FOSSE AUX LIONS - PRESENTATION DE LA VIERGE AU TEMPLE: PARALLELES ICONOGRAPHIQUES}

L'iconographie de Daniel dans la fosse aux lions, et plus particulièrement celle de l'épisode d'Habacuc lui

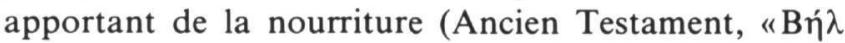

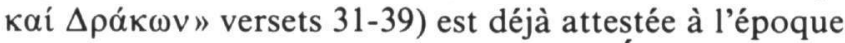
paléochrétienne. D'après les Pères de l'Église le thème symbolise la Communion. La typologie de la scène est constituée par le thème iconographique de Daniel dans la fosse aux lions, auquel est ajouté l'ange qui apporte Habacuc tenant des pains décorés de croix et un récipient. Le thème est répandu plutôt à l'époque tardo-byzantine tant dans la peinture murale que dans celle de chevalet.

L'épisode de la Vierge nourrie par l'ange (Protévangile de Jacques, VII : 1-3, VIII : 1-2) préfigurant l'Incarnation, fait partie de la scène de la Présentation au Temple à partir du XIe siècle. Quant à son iconographie, elle est composée par la Vierge et de l'ange lui donnant un pain.
Les scènes ci-dessus sont marquées d'une parenté iconographique: l'effigie volante tenant un objet, soit un pain ou un récipient. Un récipient proche de celui tenu par Habacuc caractérise l'épisode de la Vierge nourrie par l'ange dans des exemples de la Présentation au Temple du XIIe au XIVe siècle: Saint-Athanassios, Evangelistria, Saint-Sôzon, Saint-Chrysostomos à Geraki, Saint-Nicolas-tis-Rhodias de Arta. Quelquefois y est ajouté le pain décoré d'une croix.

L'Homélie de Constantin Messaritès (XIIe siècle), 'à l'occasion de la fête de la Présentation au Temple prouve les sources théologiques de l'iconographie: se référant aux préfigurations de l'Incarnation et de la Communion, Messaritès approche non seulement les épisodes en question mais aussi celui d'Élie dans le désert nourri par le corbeau et d'Isaïe par le séraphin. 